\title{
Reflets
}

Revue d'intervention sociale et communautaire

\section{Vie militaire et paternité}

\section{Karine Régimbald et Jean-Martin Deslauriers}

Volume 16, numéro 1, printemps 2010

Paternités méconnues (Deuxième partie)

URI : https://id.erudit.org/iderudit/044447ar

DOI : https://doi.org/10.7202/044447ar

Aller au sommaire du numéro

Éditeur(s)

Reflets : Revue d'intervention sociale et communautaire

ISSN

1203-4576 (imprimé)

1712-8498 (numérique)

Découvrir la revue

Citer cet article

Régimbald, K. \& Deslauriers, J.-M. (2010). Vie militaire et paternité. Reflets, 16(1), 182-211. https://doi.org/10.7202/044447ar

Tous droits réservés $@$ Reflets : Revue d'intervention sociale et communautaire, 2010

dest protégé par la loi sur le droit d'auteur. L’utilisation des services d'Érudit (y compris la reproduction) est assujettie à sa politique d'utilisation que vous pouvez consulter en ligne.

https://apropos.erudit.org/fr/usagers/politique-dutilisation/ 


\title{
Vie militaire et paternité
}

\author{
Karine Régimbald \\ Étudiante à la maîtrise, École de service social, Université d'Ottawa \\ Jean-Martin Deslauriers \\ Professeur, École de service social, Université d'Ottawa
}

\section{Introduction}

Les familles de militaires font face aux mêmes défis que l'ensemble des familles canadiennes : des préoccupations personnelles et professionnelles, le soin et l'éducation des enfants, la transmission de valeurs familiales et parfois une présence à des aînés (Drummet, Coleman et Cable, 2003). En sus de ces défis, les familles de militaires doivent composer avec des facteurs déstabilisants propres à leurs conditions de travail qui complexifient leur quotidien et influencent la façon dont les rôles et responsabilités s'y inscrivent. Plusieurs des écrits scientifiques portant sur les familles de militaires s'intéressent aux particularités de leur mode de vie (Harrison, 2006; Huebner, et collab., 2007; Drummet, Coleman et Cable, 2003; Basham, 2008; Briggs et Atkinson, 2006), mais en dépit du nombre élevé de pères militaires, peu d'études portent sur les spécificités liées à leur rôle.

Néanmoins, même si les écrits abordent généralement la situation familiale sans distinction de genre chez les parents militaires, il est justifiable d'appliquer l'essentiel de leurs données au vécu des pères, et ce, en nous basant sur le fait que les hommes comptent pour $85,4 \%$ des effectifs militaires (Park, 2008). C'est sur cette prémisse de départ que s'appuie notre décision d'aborder le rôle paternel dans le contexte de la vie militaire, et ce, à travers une recension d'écrits qui se penchent sur le sujet. L'exercice 
servira à dégager des pistes de questionnements susceptibles de conduire à des recherches ultérieures.

Nous nous pencherons d'abord sur quelques aspects de la vie militaire dans le contexte canadien, ses conditions de travail et ses incidences sur la vie familiale. Puis, nous relèverons les facteurs qui influencent chez les hommes militaires les façons de vivre leur paternité et de s'engager dans la vie de leur enfant, à la lumière des enjeux liés à la conciliation travail et vie de famille. Nous traiterons des implications des services, plus particulièrement de la pratique du travail social, s'adressant aux pères militaires et à leurs familles. Également, nous dégagerons les points de tension émergeant de l'ensemble des facteurs influant sur l'exercice de la paternité chez les militaires, notamment à la lumière des travaux de Goffman (1968) sur les institutions dites totalitaires.

\section{Conditions du travail militaire, vie de couple et vie familiale}

Plusieurs auteurs soulèvent certaines contradictions qui existent entre les exigences du système familial et l'organisation militaire en tant qu'employeur (Ryan-Wenger, 2001; Drummet, Coleman et Cable, 2003; Harrison, 2006; Harrison, 2002; Harrison et Laliberté,

"L'organisation militaire prône la cohésion des troupes et la primauté des besoins du pays sur ceux des soldats et de leur famille. » 1997; Darwin et Reich, 2006). L'organisation militaire prône la cohésion des troupes et la primauté des besoins du pays sur ceux des soldats et de leur famille. Par leur engagement, les quelque 68443 militaires des Forces régulières et les 34709 réservistes sont exposés à des absences prolongées, à des déploiements à l'étranger, à des périodes de réintégration au sein de leurs familles et à des réaffectations fréquentes. Tout cela exige d'eux, de leurs couples et de leurs familles, de grands efforts d'adaptation auxquels s'ajoute, pour les familles francophones, un facteur linguistique.

\section{Les déploiements et les séparations prolongées}

Des stages de formation, des entraînements intensifs en vue d'un déploiement ou des séjours d'observation ou de supervision dans d'autres bases militaires, voilà autant d'occasions pour les militaires d'être séparés de leurs familles. "Most army families experience annual duty-related separations, one third for more than a total of 17 weeks " 
"...le simple fait

de se préparer à voir le père quitter le domicile, de se réorganiser pendant son absence et de le réintégrer à la routine familiale à son retour occasionne plusieurs chambardements dans les habitudes familiales."
(Orthner, 2002b, dans Drummet, Coleman et Cable, 2003, p. 281). Inhérents à la carrière militaire, les déploiements constituent un important facteur de stress pour les familles. Durant cette absence, toutes les responsabilités de la maisonnée, l'entretien, les finances, l'éducation, la santé des membres de la famille, sans oublier de possibles soins aux familles étendues, incombent généralement à la mère (Drummet, Coleman et Cable, 2003; Hiew, 1992).

Selon Busuttil et Busuttil (2001), le simple fait de se préparer à voir le père quitter le domicile, de se réorganiser pendant son absence et de le réintégrer à la routine familiale à son retour occasionne plusieurs chambardements dans les habitudes familiales. Par exemple, Huebner et collab. (2007) expliquent qu'en l'absence du père, l'autre la mère devra assumer la totalité des responsabilités liées à l'éducation, telles la supervision des devoirs et l'organisation des loisirs. Il arrive aussi que les enfants s'acquittent d'un plus grand nombre de tâches. Ce nouveau partage de responsabilités aura pour conséquence de rapprocher les membres de la famille demeurés sur place.

Aux facteurs de stress liés aux séparations répétées, s'ajoutent la distance physique ainsi que le danger de plusieurs missions : les familles des soldats déployés vivront un stress proportionnel à la longueur et à la dangerosité du conflit. Lors de certains déploiements, il sera plus difficile pour le militaire d'entretenir la communication avec les membres de sa famille (Drummet, Coleman et Cable, 2003). En outre, l'attention que les médias accordent au conflit et le manque d'appui de la population à la cause peuvent engendrer plus d'inquiétude chez les familles.

Chez les enfants, les réactions face à la réorganisation familiale et à l'absence du parent militaire varient selon l'âge. Alors que les poupons peuvent avoir tendance à refuser de manger, les bambins et les enfants d'âge préscolaire peuvent afficher des comportements indiquant une régression dans leur développement et peuvent se montrer irritables (Pincus, et collab., 2008). Toujours selon ces auteurs, les enfants d'âge scolaire auront plus tendance à présenter des plaintes psychosomatiques et une mine basse alors que les adolescents se renferment davantage et sont plus à risque de développer des comportements autodestructeurs, comme 
l'utilisation de drogues et d'alcool. Pincus et collab. (2008) font remarquer que ces comportements ne sont pas inévitables, mais toutefois prévisibles dans le cadre de l'adaptation à une nouvelle situation familiale : les enfants vivent le stress d'avoir un père déployé en zone de crise.

\section{La réintégration des pères militaires à leur foyer}

Les difficultés ne se résorbent pas nécessairement avec le retour du soldat déployé. Sa réintégration au foyer constitue parfois une nouvelle adaptation pour chacun des membres de la famille.

Although reunion may be joyously anticipated, it can be as challenging as the separation (Wood, et al., 1995). Six reunion factors are of particular concern:

(a) roles and boundaries, (b) household management, (c) honeymoon effects, (d) social support, (e) parental rejection and anxiety, and (f) service member's physical and mental condition (Drummet, Coleman et Cable, 2003, p. 282).

"De plus, il arrive que les soldats déployés espèrent retrouver intacts la routine et les liens familiaux, tels qu'ils étaient au moment du départ. Pour compenser la dureté de leurs conditions de travail, il leur arrivera même de les idéaliser,... »
À cause de changements apportés à la routine, il n'est pas rare que le militaire se sente exclu à son retour. Parfois, le lien familial doit être rebâti. À ce sujet, ce discours d'un adolescent cité dans la recherche de Huebner et collab. (2007) est éloquent : «Dad was gone so you didn't have that guy-to-guy bonding. But mom had to pretty much do everything. Kind of got more attached to my mom. It was kind of a problem when dad got back because you're more attached to one parent now" (p. 19).

De plus, il arrive que les soldats déployés espèrent retrouver intacts la routine et les liens familiaux, tels qu'ils étaient au moment du départ. Pour compenser la dureté de leurs conditions de travail, il leur arrivera même de les idéaliser, ce que plusieurs auteurs nomment l'effet lune de miel : "For the husband, "long played" fantasies of the homecoming, in the face of the indefinitely prolonged, dehumanizing and depersonalizing experiences, tend to provoke exaggerated and unrealistic expectations.» (Oboler, 1987, dans Busutill et Busutill, 2001, p. 218). 
En effet, après une longue absence parsemée de crainte et d'angoisse, il est normal que l'on s'attende du parent militaire qu'il recommence à exercer ses responsabilités familiales aussitôt son retour et qu'il réponde aux besoins affectifs des siens comme il le faisait auparavant. Or, ce n'est pas toujours possible : "Fathers and mothers experiencing posttraumatic stress reactions may not be prepared for the amount and degree of need in their children.» (Caselli et Motta, 1995, dans Horton, 2005, p. 262).

Généralement, l'appui de la famille suffit pour que le soldat puisse reprendre son ancienne routine (Bowen, et collab., 2003). Toutefois, lorsqu'un soldat présente des symptômes de détresse psychologique, il n'est pas rare que la famille, la conjointe en particulier, subisse un traumatisme secondaire (Dirkzwager, et collab., 2005). L'affectation la plus commune chez les conjointes de militaires aux prises avec des difficultés d'adaptation ou encore des problèmes de santé mentale à leur retour est l'usure de compassion (Figley, 2005). Celle-ci se présente sous forme de symptômes associés à l'état de stress post-traumatique, comme des problèmes psychosomatiques, des troubles du sommeil, l'isolement social et une baisse de la qualité de la relation de couple. Rappelons néanmoins que ces résultats ne s'appliquent qu'aux familles dont un membre militaire a subi un traumatisme. Par contre, d'autres éléments de la vie militaire, susceptibles d'influer sur la famille, sont plus généralisés, parmi eux, les réaffectations fréquentes.

\section{Les réaffectations fréquentes}

Le milieu militaire exige de multiples déménagements de la part d'un bon nombre de ses employés :

Most Canadian Forces members are transferred to a new geographical location every few years, although the frequency of this practice has declined. The higher the rank a member achieves, the more transfers s/he can expect. The normal Canadian Forces career involves around 10 moves; fast-track careers involve more (Freeman, 1994, dans Harrison, 2002, p. 551$552)$. 
"Dans ce contexte de réaffectations fréquentes, les épouses sont sujettes à passer d'un emploi à l'autre, renonçant ainsi à leurs bénéfices, aux avantages sociaux, à l'ancienneté, et aux occasions de formation..."
Ces réaffectations impliquent nécessairement que les familles doivent réorganiser leur quotidien en plus de s'adapter à un nouvel environnement, surtout lors d'une affectation dans un autre pays (Drummet, Coleman et Cable, 2003). De plus, les réaffectations ont un impact négatif non négligeable sur les épouses des soldats, car elles anéantissent leur possibilité d'occuper un emploi stable. Dans ce contexte de réaffectations fréquentes, les épouses sont sujettes à passer d'un emploi à l'autre, renonçant ainsi à leurs bénéfices, aux avantages sociaux, à l'ancienneté, et aux occasions de formation (Harrison, 2002; 2006). En somme, une grande mobilité géographique entrave la stabilité d'emploi ainsi que le démontrent plusieurs auteurs (Boyle, et collab., 2001; Bielby et Bielby, 1992; Jürges, 2006).

Par contre, les enfants des militaires réagissent différemment aux réaffectations. Selon plusieurs auteurs, ils s'adaptent mieux aux changements (Horton, 2005; Ryan-Wenger, 2001; Pittman, Kerpelman et McFadyen, 2004), entre autres, à l'école où ils obtiennent souvent de meilleurs résultats que la moyenne ou se classent à tout le moins dans la moyenne générale (Strobino et Salvaterra, 2000).

Finalement, les réaffectations fréquentes font obstacle au maintien des réseaux sociaux (famille élargie, amis et communauté), lesquels rétrécissent au fil des déménagements (Harrison, 2006). Cependant, selon d'autres chercheurs, les familles de militaires s'intègrent rapidement et activement dans leur nouvelle communauté et renouvellent leurs réseaux sociaux, d'abord à travers les nouveaux collègues de travail du soldat, leurs conjoints, enfants et amis, et ensuite dans la communauté d'accueil, y compris auprès des services communautaires, tant civils que militaires (Pittman, Kerpelman et McFadyen, 2004; Drummet, Coleman et Cable, 2003).

\section{Le facteur linguistique}

La proportion de francophones et les difficultés auxquelles ils font face au sein des Forces canadiennes constituent un enjeu supplémentaire qui influence les conditions de travail des pères militaires et la vie de leur famille. Selon le Commissariat aux 
"...il arrive que le nombre et la qualité des services aux francophones soient moindres dans les régions unilingues anglophones $d u$ Canada..." langues officielles du Canada (2007), en 2005-2006, « [1]'effectif total des Forces canadiennes est composé de 27,5\% de francophones et de 72,5 \% d'anglophones ".

Malgré les efforts des Forces canadiennes, institution gouvernementale offrant le plus de cours de langue seconde au Canada, plusieurs critiques sont soulevées concernant leur niveau de bilinguisme dans l'environnement de travail. En effet, la majorité des bases militaires étant situées dans des zones géographiques unilingues anglophones, la plupart des militaires anglophones, même en ayant accès aux cours de langue seconde, ne répondent pas aux normes de bilinguisme exigées alors que les militaires francophones y répondent presque tous; ces derniers devraient ainsi obtenir plus facilement des promotions pour des postes désignés bilingues (Canada, ministère de la Défense nationale, 2006). Dans les faits, les militaires francophones ont moins accès à l'entraînement et à la formation dans leur langue, ce qui limite en quelque sorte leurs possibilités d'avancement (Tardif et Brady, 2009). Selon le Commissariat aux langues officielles (2007), seulement 35,2\% des membres du personnel militaire affecté à des postes bilingues se conforment aux exigences linguistiques émises par le comité aux langues officielles. Par ailleurs, les services personnels aux militaires et à leur famille doivent être offerts dans la langue de préférence du demandeur (Canada, ministère de la Défense nationale, 2006). Toutefois, il arrive que le nombre et la qualité des services aux francophones soient moindres dans les régions unilingues anglophones du Canada (Commissariat aux langues officielles, 2007). Dans ce contexte, il est pertinent de se demander comment s'articulent les demandes du milieu militaire et celles de la famille francophone. On peut poser l'hypothèse qu'en plus d'ajouter une pression sur les militaires francophones, ce contexte linguistique minoritaire augmente l'isolement vécu par les conjointes de ces pères lorsqu'ils sont déployés.

\section{Le travail militaire et la vie de couple}

Compte tenu de l'ensemble des données qui précèdent, il nous faut reconnaitre qu'au chapitre de la vie de couple et de la structure familiale, le mode de vie militaire entraîne des défis autres que ceux 
"...les soldats divorcés sont beaucoup moins susceptibles d'obtenir la garde légale de leurs enfants, et ce, à cause de leur mode de vie, de leur situation conjugale instable et de leurs échecs amoureux." rencontrés dans le reste de la population (Drummet, Coleman et Cable, 2003; Corriveau, 2007). Les séparations fréquentes sont une grande source de frustration, au même titre que plusieurs autres irritants qui peuvent souvent entraîner un divorce : "Job demands such as combat deployments, frequent relocations, separations from family, long hours, and stressful job tasks that involve physical and mental danger constitute another divorce risk. " (Adler-Baeder, Pittman et Taylor, 2005, p. 92-93). En effet, une étude américaine démontre que l'on divorce et se remarie davantage chez les militaires que dans la population civile. De plus, les soldats américains sont aussi plus souvent pères d'enfants dont ils n'ont pas la garde (Adler-Baeder, Pittman et Taylor, 2005). Selon ces chercheurs, les soldats divorcés sont beaucoup moins susceptibles d'obtenir la garde légale de leurs enfants, et ce, à cause de leur mode de vie, de leur situation conjugale instable et de leurs échecs amoureux. Fréquemment réaffectés à cause du travail, il leur devient difficile de maintenir des relations de qualité avec leurs enfants.

Autre aspect de la vie de couple en relation avec le travail militaire : les particularités de ce dernier peuvent contribuer à la violence à l'égard des femmes (Harrison, 2002). Les conjointes de militaires seraient jusqu'à cinq fois plus sujettes à vivre de la violence conjugale que leurs consœurs dans le civil (Mercier, 2000). Selon Harrison (2006), l'institution militaire, toute consciente est-elle de l'adaptation exigée des familles quant aux responsabilités familiales, exerce quand même une forme d'oppression sur les conjointes. L'isolement et la dépendance financière causés par les réaffectations fréquentes en seraient la principale cause (Harrison, 2006).

Bref, pour les militaires, la vie de couple et la structure familiale sont grandement influencées par les exigences rattachées à leur métier de soldat, principalement celles résultant des déploiements et des séparations prolongées, auxquelles s'ajoutent un possible sentiment d'exclusion lors de la réintégration et des réaffectations fréquentes. Le travail inhérent au métier de soldat peut aussi avoir des répercussions sur la santé mentale des personnes qui s'y engagent. 


\section{La santé mentale des pères militaires}

Les soldats subissent parfois des séquelles des dangers auxquels les expose leur travail (Drummet, Coleman et Cable, 2003). À leur retour de mission, plusieurs souffrent de problèmes de santé physique et mentale. L'état de stress post-traumatique est l'affection la plus souvent diagnostiquée chez les militaires (Creamer et Forbes, 2004). Ce diagnostic désigne un trouble de santé mentale précis : il se retrouve spécifiquement chez les individus ayant été exposés à un événement mettant leur vie ou leur sécurité en danger (Van der Kolk,Van der Hart et Burbridge, 2002). Selon ces auteurs, une personne en état de stress post-traumatique revivra la situation traumatique de diverses manières, soit par des flashbacks, des rêves éveillés, des cauchemars, des émotions ou des expériences sensorielles. Par la suite, la personne affectée aura tendance à répondre à ces intrusions du passé par le refoulement ou par l'engourdissement de sa douleur par toutes sortes de moyens; les médicaments, les drogues, l'alcool sont les échappatoires les plus souvent utilisées (Creamer et Forbes, 2004).

Les symptômes associés à cet état de stress post-traumatique entravent la capacité d'un soldat à répondre aux exigences de la vie de famille. Par exemple, une des réactions les plus courantes au trauma est l'hypervigilance : elle peut mener à des sautes d'humeur, à des explosions de colère et d'agressivité (Van der Kolk, Van der Hart et Burbridge, 2002). Ces symptômes vont troubler et perturber la famille du soldat à son retour au foyer.

Le risque de suicide est plus élevé chez les personnes souffrant d'un trouble de la santé mentale, particulièrement chez celles qui ont vécu un événement traumatique, comme une agression sexuelle, ou qui ont tué ou blessé d'autres personnes (Belik, et collab., 2009). En revanche, le taux de suicide chez les hommes membres des Forces canadiennes est plus bas que celui des hommes de la même tranche d'âge de la population générale (Canadian Forces Health Services Group, 2010). Cependant, les données de Statistique Canada servant à comparer la population militaire dans cette étude ne sont disponibles que pour 2005, et avant. Par la suite, le nombre de suicides chez les soldats canadiens 
"Il y a donc lieu d'affirmer que certains pères militaires, surtout ceux pratiquant des métiers de combat, sont à haut risque de présenter des idéations et des gestes suicidaires au retour d'un déploiement en zone hostile." augmente, particulièrement entre 2006 et 2008, au point de rejoindre le taux de suicide des hommes canadiens enregistré en 2005. Le risque d'une tentative de suicide serait plus élevé chez les militaires canadiens ayant subi une agression sexuelle que chez ceux exposés au combat (Belik, et collab., 2009). Il serait par contre plus élevé chez les soldats ayant tué ou blessé autrui ou ayant été torturés (Belik, et collab., 2009).

Cette nuance est importante puisqu'elle indique que le fait de travailler pour les Forces canadiennes ou encore d'être déployé en zone de conflit ou de crise humanitaire ne constitue pas en soi un risque de suicide, à moins d'y subir un traumatisme ou de développer des problèmes de santé mentale à la suite de cette exposition. Même si elles ne permettent pas de connaitre avec exactitude les causes et les facteurs de risque associés au suicide chez les militaires canadiens, ces données indiquent que depuis le début de la guerre en Afghanistan, plus de soldats canadiens sont exposés au danger, les rendant ainsi plus à risque de développer des problèmes de santé mentale, et donc plus à risque de se suicider. Il $\mathrm{y}$ a donc lieu d'affirmer que certains pères militaires, surtout ceux pratiquant des métiers de combat, sont à haut risque de présenter des idéations et des gestes suicidaires au retour d'un déploiement en zone hostile.

\section{Valeurs militaires, masculinité et paternité}

Dans la section qui suit, nous verrons comment la vie militaire entraîne une socialisation à un schème de valeurs particulier, lequel comprend une conception traditionnelle de la façon dont les hommes doivent se comporter. Cet effet de la vie militaire a son influence sur l'exercice de la paternité.

Pour traiter de cet aspect, rappelons que la socialisation se définit comme étant :

[...] le processus par lequel la personne humaine apprend et intériorise tout au cours de sa vie les éléments socioculturels de son milieu, les intègre à la structure de sa personnalité sous l'influence 
d'expériences et d'agents sociaux significatifs et par là s'adapte à l'environnement social où elle doit vivre (Rocher, 1992, p. 132-133).

Le processus de socialisation se déroule en deux étapes. D'abord, la socialisation primaire a lieu dans l'enfance à travers deux institutions principales, soit la famille et l'école.Vient ensuite la socialisation secondaire à travers le champ professionnel et les réseaux sociaux et politiques durant l'âge adulte (Dubar, 2000). On peut aussi parler de resocialisation lorsque l'individu est soumis à une nouvelle institution comme l'armée, la prison ou l'asile, qui remet profondément en cause les principes et normes acquis durant l'enfance (Macé, 2002). Selon Schehr (2005), « [il] en résulte une socialisation militaire plus "forte" au sens où celleci est ressentie comme une véritable rupture avec les formes de socialisation antérieures » (p. 207).

Pour Dubar (2000), en plus du processus d'apprentissage et de reconnaissance des normes et des valeurs de l'environnement social dans lequel on évolue, " [s]e socialiser, c'est assumer son appartenance à des groupes, c'est-à-dire, prendre personnellement en charge ses attitudes au point où elles guideront largement sa conduite sans même qu'on s'en rende compte " (Dubar, 2000, p. 32).

\section{"La socialisation secondaire issue de la vie militaire influe grandement sur les perceptions qu'ont les soldats de la masculinité. »}

\section{Valeurs militaires et masculinité}

La socialisation secondaire issue de la vie militaire influe grandement sur les perceptions qu'ont les soldats de la masculinité. Celle-ci pourrait être définie comme la perception qu'ont les individus et les sociétés des traits physiques et de caractère ainsi que des rôles et des tâches associés au fait d'être de sexe masculin. Or, la société occidentale associe plusieurs attitudes à la masculinité, définissant ainsi les stéréotypes masculins (David et Brannon, 1976, dans Kahn, 2009, p. 57) :

- Men should be emotionally stoic and deny vulnerability;

- Men should be preoccupied with work, status, achievement, and success; 
"Ces caractéristiques stéréotypées reflètent bien la culture masculine dont l'institution militaire est empreinte (Winslow et Dunn, 2002). Les Forces canadiennes misent sur l'agressivité, l'orgueil, la compétitivité et la force physique pour mener à bien leurs opérations. »
- Men should be forceful and interpersonally aggressive;

- Men should reject anything associated with femininity.

On peut facilement observer ces attitudes dans le milieu militaire, particulièrement lors de la resocialisation engendrée par le cours de qualification de base, appelé communément camp des recrues, et des périodes d'exercice intensives en pré-déploiement où les stéréotypes sexuels sont renforcés. On peut comparer le cours de qualification militaire de base à la socialisation secondaire puisque les jeunes soldats évoluent non seulement dans un contexte scolaire d'apprentissage, mais ils doivent aussi vivre ensemble, telle une famille, et partager une grande part de leur intimité (Schehr, 2005). Cette resocialisation s'ajoute à la socialisation primaire qui tend elle-même à reproduire les stéréotypes sexuels, omniprésents dans la culture militaire (Levant et Pollack, 1995) : "The military uses its "socially constructed polarity" between masculine and feminine in order to use masculinity as the "cementing principle" which unites military men in order to distinguish them from non-military men and women." (Harrison et Laliberté, 1997, p. 36).

Ces caractéristiques stéréotypées reflètent bien la culture masculine dont l'institution militaire est empreinte (Winslow et Dunn, 2002). Les Forces canadiennes misent sur l'agressivité, l'orgueil, la compétitivité et la force physique pour mener à bien leurs opérations. D'autres valeurs comme la force psychologique, l'endurance, l'ambition professionnelle, la hiérarchie, le leadership, le courage, l'honneur, la persévérance, la loyauté et le sens du devoir sont fortement appuyées par les Forces canadiennes (Canada, ministère de la Défense nationale, 2008a) : "The male identity of the organization is strongly evident through sexualised behaviours and norms, such as male attitudes of paternalism, sexist male talk, joking and innuendo, pinups and calendars, and sexual harassment, in the day-to-day environment of work." (Davis, 1997, dans Winslow et Dunn, 2002, p. 651).

La vie émotive est d'ailleurs une des facettes que le soldat réprime, de manière à ne pas ébranler la masculinité traditionnelle jugée nécessaire au combat (Jelusic, 2005). L'auteur explique que les émotions, d'abord nommées et intériorisées par tous, sont 
canalisées en des objectifs de combat au nom desquels le groupe tend vers un même but, créant ainsi le sentiment d'unité et de cohésion. Les émotions qui ne seront pas dirigées vers le combat sont aussi ritualisées lors de cérémonies commémoratives où l'on permet leur expression de manière à conserver l'équilibre dans la troupe. Ainsi, un soldat qui exprimerait ses émotions en dehors des rituels appropriés sait qu'il cause le déséquilibre dans son groupe. Il sera puni, car on dit de lui qu'il risque sa vie et celle de ses coéquipiers (Jelusic, 2005).

Army work is about the defense of political interests home and abroad, with soldiers regularly being expected to deal with danger, death, and suffering on a large scale. They work in these environments with due care to their own physical safety, but without reflecting upon the emotional effect of the environment upon themselves. There is no place for emotion in such work, and there is certainly no space to think about the emotional impact of episodes during tours of duty. [...] Emotion has no meaning or significance in such a culture (Briggs et Atkinson, 2006, p. 52).

Ce code de conduite prescrit clairement une conception de la masculinité qui, par extension, est susceptible de teinter la définition de la paternité. En effet, les hommes qui deviennent pères agissent en conformité avec ce qu'ils estiment être un bon modèle masculin (Deslauriers, 2009). Or, si le schème de référence intégré est nettement stéréotypé, adopter des comportements ou attitudes considérés féminins dans l'ensemble du contexte de vie militaire peut constituer un défi dans l'exercice de la paternité. D'autre part, tous les militaires n'ont pas à se conformer au même degré à ces stéréotypes sexuels. On peut distinguer les métiers de combat, où l'utilisation délibérée des stéréotypes sexuels est considérée comme nécessaire pour susciter l'agressivité et refouler l'émotivité, des métiers techniques ou professionnels qui se retrouvent aussi dans la sphère civile : "We argue that the combat arms are far more removed from civilian society. As a result they 
emphasize the values and attitudes of the traditionally male-oriented military organization and, in particular, masculine models of the warrior. " (Winslow et Dunn, 2002, p. 642).

Quant aux membres occupant des postes de soutien ou administratifs, ils sont moins susceptibles d'être soumis à la pression provoquée par cette conception de la masculinité, à tout le moins pas de façon aussi contraignante.

\section{Valeurs militaires et paternité}

L'exercice de la paternité fait référence aux comportements et attitudes adoptés par le père envers ses enfants. Plus précisément, Lamb (2000) définit la paternité comme étant la relation qu'un père développe avec son enfant, les influences de cette relation sur son développement et son impact sur l'enfant, sur le père lui-même et sur la dynamique familiale. L'auteur rappelle que le concept de la paternité n'est pas statique et évolue dans le temps et au fil des expériences et des transformations sociales et culturelles.

Ainsi, l'engagement paternel peut prendre plusieurs formes et rapporter plusieurs bénéfices à tous les membres de la famille (Smith, 2004; Forget, 2009; Flouri et Buchanan, 2002; Featherstone, 2004; Caragata et Miller, 2008; Allen et Daly, 2007; Dubeau, Clément et Chamberland, 2005). Dans des études récentes portant sur la paternité et la promotion de l'engagement paternel, un groupe de chercheurs a développé une définition en plusieurs dimensions de l'engagement paternel, à savoir, « le père pourvoyeur, le père responsable, le père en interaction, le père qui prend soin, le père affectueux, le père évocateur et le père citoyen " (Forget, 2009, p. 82). Selon cette perspective, « le niveau d'engagement paternel résulte de l'interaction dynamique de facteurs relevant à la fois de caractéristiques du père, des enfants, de la mère et du contexte social " (Forget, 2009, p. 82). Un aspect important de cette conception de l'engagement paternel veut qu'il s'exerce autant en présence de l'enfant qu'à distance, ce qui évite de le réduire au simple calcul du temps passé auprès de l'enfant (Featherstone, 2004; Forget, 2009). 
"Reconnaissons toutefois que la négation de la vulnérabilité et le contrôle des émotions appris dans les Forces canadiennes puissent teinter l'exercice de la paternité chez ces hommes. »
Brooks (1998) explique que l'exercice de la paternité est en majeure partie déterminé par la socialisation primaire d'un homme. Les comportements et attitudes associés à sa paternité refléteraient le modèle de père qu'il a eu. À moins d'une prise de conscience de lacunes dans cette socialisation et une volonté consciente de ne pas reproduire le modèle reçu, un père serait plus influencé par son enfance dans l'exercice de sa paternité que par les valeurs acquises par la suite. Par contre, la socialisation secondaire militaire s'opère en rupture avec les formes antérieures de socialisation : l'organisation se substitue en quelque sorte à la famille dans ce processus (Schehr, 2005). Certains pères pourraient donc intégrer le modèle militaire et le reproduire dans l'exercice de leur rôle, surtout si l'expérience s'est avérée plus positive que celle de la socialisation primaire qui remonte à leur enfance (Brooks, 1998).

Cependant, les recherches actuelles ne permettent pas d'établir jusqu'à quel point les valeurs militaires et les valeurs familiales influencent l'exercice de la paternité chez les militaires canadiens. Dans cette optique, il serait intéressant que la recherche vérifie cette hypothèse : plus la socialisation secondaire aux valeurs militaires sera intériorisée par le père, plus il aura tendance à transmettre ces valeurs à ses enfants, entre autres, la discipline personnelle et le respect de la hiérarchie. Par exemple, discipline, rigueur, adaptabilité au changement seront exigées des enfants sans possibilité pour eux de négocier ou d'exprimer leur point de vue sur une règle précise.

Reconnaissons toutefois que la négation de la vulnérabilité et le contrôle des émotions appris dans les Forces canadiennes puissent teinter l'exercice de la paternité chez ces hommes. Un père militaire pourrait donc avoir tendance à négliger sa propre vulnérabilité physique et affective ainsi que celle de ses enfants afin de leur transmettre des valeurs d'invulnérabilité et de contrôle de soi. Les hommes au style parental traditionnel se contenteraient plutôt de jouer un rôle de pourvoyeur et de représentant de la discipline auprès des enfants tout en déléguant les fonctions affectives et relationnelles à la mère (Brooks, 1998). 
Par ailleurs, il faut se demander comment, dans leur vie personnelle, certains pères militaires parviennent à se distancier de la forte pression exercée par la vie militaire et à fonder leur engagement paternel sur le modèle qu'ils ont connu enfants. La question mérite d'être examinée pour le regard qu'elle propose sur l'expérience de soldats qui parviennent à vivre leur paternité à l'abri des influences et des aléas de leur métier.

\section{Soutien aux familles au sein des Forces canadiennes}

Les Forces canadiennes disent reconnaître l'importance de la famille dans l'accomplissement de ses engagements envers le pays (Canada, ministère de la Défense nationale, 2008c) et affirment s'engager à travailler avec les familles et les communautés civiles à améliorer le mode de vie militaire. L'organisation militaire demande aux familles, comme à ses membres, de s'adapter à ses valeurs, ses exigences et ses politiques et elle leur fournit une gamme importante de services pour y parvenir.

Mesures de soutien et services sociaux destinés aux pères militaires et à leur famille

Parmi les mesures adoptées afin de les soutenir, les pères militaires bénéficient de plus de jours de congé pour assumer leurs responsabilités familiales; leur horaire de travail est assoupli pour leur permettre d'assurer une présence à la maison aux moments où les enfants en ont le plus besoin (Bass, et collab., 2009; Tremblay, Amherdt et De Sève, 2003; Voydanoff, 2004; Caragata et Miller, 2008).

De plus, les Forces canadiennes ont mis en place un système de santé réservé à leurs membres et assuré par différents professionnels, médecins, dentistes, spécialistes, travailleurs sociaux, psychiatres, psychologues, aumôniers, infirmiers ou autres (Canada, ministère de la Défense nationale, 2008b). Ce système répond à l'idéologie voulant qu'un soldat soit un investissement pour les Forces.

The culture is hierarchical, protective of soldiers and their family members, and by their own description, 
wary of the motives of outside groups offering services, even on a pro bono basis. Military leaders have conveyed to SOFAR personnel the fear that allowing an outside group to deliver services would indicate a failure on the part of the military to provide sufficient services for their own. Any group of psychologists seeking to provide services to the military must be prepared for a degree of difficulty in gaining access to the system (Darwin et Reich, 2006, p. 482).

À ces services professionnels, s'ajoutent ceux destinés aux familles des militaires et qui sont dispensés dans des centres de ressources. Situés dans la plupart des bases, ces centres voient à l'aspect social de la vie des militaires, à l'équilibre familial et au soutien psychologique et social afin que la famille soit en mesure de continuer de soutenir les efforts de guerre du militaire, ou du moins, de ne pas les entraver (Pittman, Kerpelman et McFadyen, 2004).

Everything in the military environment is directed toward the mission of protecting and defending the country. [...] Retention in the military is in part based on the health and happiness of the families. In addition, if the military member can stay focused on their military job while in the field, it would enhance their readiness. If the children could stay focused on their job at school, the family could also be more mission ready (Horton, 2005, p. 264).

Ces centres de ressources tentent d'inclure les pères dans leurs activités et interventions, même si leur clientèle principale reste les conjointes et les enfants (Centre de ressources famille-militaire de Valcartier, 2009). Par contre, leur mandat reste flou, car leurs objectifs semblent viser davantage la gestion du stress associé au mode de vie militaire pour que la famille puisse à son tour " contribuer à l'efficacité opérationnelle des Forces canadiennes " (Services de soutien au personnel et aux familles des Forces canadiennes, 2010). 


\section{Le travail social auprès des militaires et de leurs familles}

$\mathrm{Vu}$ la place importante qu'elles lui accordent dans la gamme de leurs services, il importe de connaitre la vision des Forces canadiennes sur le travail social et le rôle qu'elles lui attribuent. Elles demandent au travailleur social d'accomplir les tâches suivantes (Canada, ministère de la Défense nationale, 2008d) :

- Fournir des services d'intervention clinique directement à des militaires canadiens et à des membres de leurs familles;

- Contribuer au règlement de problèmes personnels de manière à éviter des mesures relatives à la carrière;

- Consulter et conseiller des commandants et des superviseurs au sujet des problèmes personnels de membres de l'unité;

- Seconder des officiers responsables des questions de carrière en menant des enquêtes et en rédigeant des rapports sur des problèmes personnels;

- Offrir des programmes de prévention et de réadaptation dans les domaines du stress lié au prédéploiement et au post-déploiement, du suicide et de la violence familiale.

Daley (2003) explique que les objectifs du travail social militaire sont de contribuer au développement de la capacité du soldat à se remettre de l'expérience de la guerre. De plus, les travailleurs sociaux conseillent les autorités militaires dans l'adoption de mesures permettant d'améliorer la qualité de vie de leurs membres et de leur famille tout en tenant compte des contraintes inhérentes au travail militaire. La tâche des travailleurs sociaux est aussi de s'assurer que les problèmes familiaux se règlent le plus rapidement possible afin que le père puisse mieux se concentrer sur son travail. On peut se demander si ces pratiques qui font passer les objectifs de l'institution avant ceux des personnes sont conformes aux codes de déontologie des travailleurs sociaux. Par ailleurs, le fait de devoir conseiller les superviseurs sur l'état de santé et sur les problèmes familiaux des membres des Forces canadiennes pour déterminer s'ils sont aptes ou non à combattre est tout aussi discutable. 
Comme plusieurs auteurs ont affirmé que les Forces canadiennes ont leur propre culture (Harrison, 2006; Winslow et Dunn, 2002; Jelusic, 2005), l'analyse des politiques sociales et des services sociaux qu'elles mettent en place permet d'en faire ressortir les valeurs principales.

Harrison (2006) juge que les Forces canadiennes perçoivent le soldat comme un investissement pour le pays. Elle explique que l'organisation militaire est prête à mettre en branle une panoplie de services sociaux et d'autres moyens coercitifs, voire punitifs, pour garder, ou ramener, ses membres sur le droit chemin, c'est-à-dire physiquement et psychologiquement aptes au combat.

Les Forces canadiennes font la promotion d'un modèle familial plutôt traditionnel où le père est perçu comme jouant principalement un rôle de pourvoyeur dans la famille, alors que la mère se voit offrir des activités et services familiaux au centre de ressources pour familles de militaires. Par contre, il faut noter que cet état de fait n'existe pas qu'au sein des Forces canadiennes : partout ailleurs, les services destinés aux familles ont encore tendance à s'adresser aux mères et à percevoir le père comme un problème (au pire) et à voir sa participation comme un bonus (au mieux) (Davies, et collab. 2009; Featherstone, 2004; Villeneuve, 2009).

\section{Questionnements et pistes de recherche émergeant de la recension des écrits}

Plusieurs questionnements émergent de notre exposé de facteurs affectant l'exercice de la paternité chez les militaires. Un enjeu crucial émane des contradictions existant entre la situation familiale des pères et le milieu de travail. Nos questionnements amènent aussi à faire le point sur l'évolution des conditions d'exercice de la paternité au sein des Forces canadiennes et enfin, à réfléchir sur le rôle des travailleurs sociaux auprès des pères militaires et de leur famille. 
Contradictions entre la situation familiale et le milieu de travail des pères militaires

L'enrôlement dans les Forces canadiennes demande au futur soldat des sacrifices importants, et ce, aux dépens de sa vie privée et familiale (Canada, ministère de la Défense nationale, 2008a). L'engagement des soldats canadiens à servir le pays avant euxmêmes constitue probablement la source des contradictions qui marquent le plus le vécu des militaires. Dans le civil, il arrive aussi qu'il soit exigé de faire passer le travail avant soi-même et, par extension, avant ses obligations familiales, mais notre recension d'écrits évoque bien l'intensité de la symbiose qui se crée entre le militaire et l'institution des Forces canadiennes qui exige de ses membres l'oubli ou la perte de soi, qui leur impose des exercices menant à une resocialisation et qui leur impose un système de valeurs. Ces phénomènes s'apparentent à ce que Goffman (1968) identifie comme une institution totalitaire, ou totale, qui est « $[\mathrm{u}]$ $\mathrm{n}$ lieu de résidence et de travail où un grand nombre d'individus, placés dans la même situation, coupés du monde extérieur pour une période relativement longue, mènent ensemble une vie recluse dont les modalités sont explicitement et minutieusement réglées » (p. 45). Les travaux de Goffman démontrent que pour atteindre un haut degré de cohésion, il faut briser l'individu afin de saper tout questionnement sur la règle, la hiérarchie, l'autorité et l'amener à faire un avec l'institution. Spécialement en contexte militaire, les intérêts du groupe transcendent la valeur de l'individu qui peut être mobilisé à tout moment (Grossman, 1995, dans Harrison, 1997). À titre d'exemple, Harrison (1997, p. 30) décrit le camp d'entraînement de base durant lequel :

[...] les aspirants échangent leur ancienne identité pour l'uniforme, la coupe de cheveux et les tâches journalières de l'armée. Ils sont humiliés, privés de leurs droits et dépouillés des réalisations de leur vie antérieure en se faisant répéter continuellement que rien de ce qu'ils ont fait avant le camp d'entraînement n'est important. Le vide ainsi créé dans l'amour-propre de l'aspirant par ce harcèlement est ensuite comblé par la nouvelle 
identité du "prêt au combat " fournie par l'armée. Peu à peu, les entraîneurs militaires remplacent les agressions verbales par de légers renforcements positifs: à leur admission, les aspirants auront appris à être fiers d'eux-mêmes selon des critères propres à l'armée.

Précisons que la vie de militaire ne se résume pas toujours à cette vie de reclus. Lorsqu'ils ne sont pas déployés, les militaires rejoignent leurs proches le soir venu, ce qui permet de limiter le caractère aliénant de l'institution, des liens étant entretenus à l'extérieur du milieu de travail qualifié de totalitaire. À l'appui de l'exemple du personnel d'un milieu psychiatrique, Goffman (1968) observe qu' " en maintenant à l'extérieur leurs liens familiaux, les membres du personnel demeurent généralement intégrés à la communauté extérieure et échappent à la tendance enveloppante de l'institution totalitaire " (p. 53).

Ainsi, bien qu'ils doivent se soumettre à un schème de référence rigide dans le cadre de leur travail, les pères militaires disposent d'une marge de liberté dans la façon de se définir à l'extérieur des Forces canadiennes. En effet, bien que cette institution impose jusqu'à un certain point une resocialisation aux personnes qui s'y engagent, celles-ci peuvent toujours régir leur vie personnelle selon leurs valeurs personnelles, notamment, en faisant place à l'écoute, à la communication dans la famille et en s'accordant un espace pour leur vie intérieure. Donc, il faut à la fois reconnaître la très grande influence de l'institution et considérer la puissance des liens familiaux qui agissent en contrepoids (Goffman, 1968).

Par ailleurs, malgré ses efforts pour constituer un milieu isolé du reste de la société, l'institution militaire reste néanmoins influencée par les institutions sociales, communautaires, politiques ou culturelles. Bon gré mal gré, elle doit tenir compte des nouvelles normes concernant la conciliation travail-famille : les soldats eux-mêmes la revendiquent de plus en plus. Néanmoins, Dulac (2009) affirme que malgré ces nouvelles tendances, la culture du milieu d'emploi joue un rôle non négligeable dans les pratiques entourant ladite conciliation. Il explique que même si certains gouvernements mettent en place des lois obligeant les 
employeurs à favoriser une meilleure conciliation travail-famille, plusieurs obstacles rendent leur utilisation difficile, entre autres, la culture de l'entreprise, la perception du rôle de père (celle de l'employé autant que de sa conjointe) et la pression sociale. En effet, la cohésion des troupes et les attitudes masculines stéréotypées sont telles que même si l'institution est mise dans l'obligation d'accorder à un père un congé parental, ce père peut être mal perçu par ses coéquipiers ou même par ses supérieurs, quand ce n'est pas lui-même qui juge injustifiable de l'utiliser (Bedford, 2009).

\section{Évolution des conditions d'exercice de la paternité au sein des Forces canadiennes?}

On remarque que les Forces canadiennes continuent de valoriser le modèle traditionnel de la famille dans le soutien de ses troupes. Au temps des grandes guerres et de la conscription, les jeunes hommes qui s'enrôlaient pour combattre étaient célibataires (Cotton, 1981).Aujourd'hui, la plupart des soldats canadiens sont mariés ou en union de fait et ont des enfants (Truscott et Dupre, 1998). Pourtant, les conditions dans lesquelles doivent évoluer les militaires exigent des épouses à être toujours prêtes à servir à leur façon, à assumer l'ensemble des responsabilités parentales dès qu'une urgence nationale monopolise leur conjoint. À l'époque des deux grandes guerres, l'homme jouait le rôle de pourvoyeur dans la famille. Or, la société contemporaine véhicule des valeurs d'égalité et de partage des responsabilités parentales au sein des familles. Les militaires se retrouvent encore une fois coincés entre les valeurs sociales contemporaines qui demandent aux pères d'accorder la priorité à la famille et les valeurs traditionnelles de l'organisation militaire qui les considère encore comme des pourvoyeurs et des agents de socialisation des enfants (Dubeau, Devault et Forget, 2009).

La définition de l'engagement paternel comprend désormais une dimension expressive, laquelle implique un lien intime avec l'enfant de même que la dispensation de soins de base (Lamb, 2000). Les hommes dont la masculinité est restée stéréotypée arrivent difficilement à répondre à ces nouvelles attentes. Il 
devient alors important d'examiner ce que les Forces canadiennes mettent en place pour permettre à leurs membres de remplir leurs obligations familiales et de se reconnaitre dans la société canadienne contemporaine.

Un des moyens qu'a trouvés l'organisation militaire pour atténuer les tensions entre la citoyenneté moderne et la vie militaire a été d'isoler les soldats plutôt que de faire des compromis sur le plan des sacrifices personnels exigés d'eux. De plus, en chapeautant elle-même les services médicaux, psychosociaux ou communautaires destinés aux soldats ou aux familles, il est évident que l'organisation militaire conserve un très grand contrôle sur le type d'intervention qui leur est offert. Comme le décrit si bien Harrison (2006), les quartiers d'habitations militaires, les déménagements fréquents et la dispensation de services exclusivement par des organismes approuvés par les Forces canadiennes, quand ils ne sont pas militaires eux-mêmes, sont autant de facteurs qui contribuent à la reproduction des valeurs de sacrifice des soldats et de leur famille et, par extension, à la propagation des problèmes sociaux qui en découlent.

Cette recension des écrits permet de formuler une critique majeure à l'intention des Forces canadiennes : bien que nombreux, les services offerts aux militaires et aux familles sont toujours orientés vers l'adaptation du soldat et de sa famille aux exigences de l'institution. De par son engagement auprès de la famille, l'organisation militaire donne même l'impression de se substituer au père dans la prise en charge de ses responsabilités familiales et de les déposséder sur ce chapitre.

Certaines mesures pourraient être prises pour s'assurer qu'en dehors du contexte de la guerre, les soldats bénéficient d'une plus grande souplesse pour veiller aux besoins de leur conjointe, de leurs enfants et aussi de leurs aînés. Si la structure hiérarchique rigide, la masculinité stéréotypée, de même que les réaffectations fréquentes et la ritualisation des émotions sont des facteurs cruciaux au bon fonctionnement des troupes, n'y aurait-il pas d'autres façons de favoriser la citoyenneté et de défendre les valeurs canadiennes? 


\section{Le rôle de travailleurs sociaux auprès des pères militaires et de leur famille}

Tel que pratiqué actuellement, le travail social militaire pose quelques problèmes éthiques. D'abord, sur le plan de la philosophie d'intervention au sein d'une institution présentant plusieurs caractéristiques se rapprochant de celles qui sont dites totalitaires (Goffman, 1968), comment demeurer critique, libre de son jugement professionnel? Sur le plan de la pratique, un enjeu se pose également. Le travailleur social et la personne auprès de qui il intervient ont le même employeur et le travailleur social doit rendre compte de son évaluation au supérieur immédiat de cette personne, ce qui pose un problème de confidentialité. On peut aussi remettre en question le pouvoir accordé au travailleur social qui doit décider, à la suite d'une évaluation tout objective soit-elle, si un soldat est apte ou non à aller au front. Le jugement du travailleur social sur la capacité pour un soldat d'être déployé ou non peut devenir une question de vie ou de mort (Rotter et Bojeva, 1999). Finalement, non seulement les travailleurs sociaux militaires doivent-ils composer avec le conflit entre le bien-être individuel et familial des soldats et le bon fonctionnement de l'institution militaire, mais un autre enjeu éthique les touche: la promotion de la paix ne fait-elle pas partie des valeurs fondamentales du travail social? Dans le monde militaire, les travailleurs sociaux font face à cette contradiction entre la promotion de la paix, dont la famille unie est un puissant symbole, et l'inhumanité de la guerre (Verschelden, 1993). En effet, le rôle d'un travailleur social ne serait-il pas de promouvoir l'humanité avant tout? Les institutions en faveur de la guerre aiment rappeler que pour atteindre la paix, on doit souvent passer par la guerre. Verschelden (1993) soutient qu'il est du devoir des travailleurs sociaux de s'opposer à la guerre.

D'un autre côté, les travailleurs sociaux militaires occupent une position clé au sein des Forces canadiennes :à titre d'experts, ils sont consultés sur le bien-être des troupes. De plus, ils peuvent formuler certaines recommandations pour que les Forces canadiennes respectent leur engagement envers les familles (Daley, 
2003). En somme, de par leur position, les travailleurs sociaux peuvent favoriser l'humanisation de l'organisation militaire, en autant que faire se peut, tout en apportant soutien et acceptation aux soldats et à leur famille. Des recherches plus approfondies sur le travail social en contexte militaire s'avèrent donc importantes pour enrichir la réflexion sur les contradictions qui le soustendent et pour dégager des voies possibles vers des pratiques plus congruentes avec ses valeurs et son éthique.

\section{Conclusion}

La recension des écrits sur les facteurs influençant l'exercice de la paternité chez les pères militaires rend compte des nombreux défis qui se présentent à eux et à leur famille et dresse un portrait complexe des éléments contextuels et individuels qui touchent la façon dont ces pères arrivent à jouer leur rôle. Notamment, plusieurs exigences envers les soldats, même considérées comme fondamentales par l'institution militaire, vont à l'encontre des facteurs qui facilitent l'exercice de la paternité. Même si des services sociaux et de santé sont déployés par les Forces canadiennes pour soutenir la famille, leur but premier est d'assurer la pleine efficacité du militaire dans l'exercice de son métier. Cela dit, est-il envisageable que l'engagement des Forces canadiennes auprès des familles de militaires passe non seulement par l'offre de services leur assurant du soutien, mais aussi par la mise en place de mesures facilitant l'investissement et l'engagement du soldat lui-même dans sa famille?

Pour les travailleurs sociaux œuvrant auprès des militaires et de leur famille, enrôlés eux-mêmes ou issus du civil, il leur importe de répondre aux questions éthiques soulevées par notre recension d'écrits. Les exigences requises pour un emploi de travailleur social auprès des militaires canadiens (Canada, ministère de la Défense nationale, 2008d) invitent à une réflexion de la part des ordres professionnels, notamment sur certaines procédures et au chapitre du respect de la confidentialité. 
Les travailleurs sociaux employés par les Forces canadiennes peuvent également se servir de leur expertise pour militer en faveur de la reconnaissance du rôle paternel. Les travailleurs sociaux ont un rôle clé à jouer dans cet exercice, soit de s'attaquer autant aux stéréotypes militaires associés à la masculinité qu'aux conditions de vie que l'organisation exige. Ces traditions obligent les militaires à se conformer au modèle familial traditionnel et les placent en contradiction avec des valeurs modernes prônées dans la société civile. À l'opposé, la recension des écrits fait ressortir que les militaires qui continuent à avoir des liens dans la communauté civile réussissent mieux à naviguer entre les deux systèmes de valeurs et, donc, à exercer leur paternité selon les représentations auxquelles ils adhèrent.

Cette recension des écrits permet enfin de voir que, tout influencé que soit le père par la vie militaire dans laquelle il s'investit, son rôle peut être facilité par des pressions externes susceptibles d'apporter des changements souhaitables au sein de l'institution.

\section{Bibliographie}

ADLER-BAEDER, Francesca, Joe F. PITTMAN et Lisa TAYLOR (2005). " The Prevalence of Marital Transitions in Military Families ", Journal of Divorce and Remarriage, Vol. 44, No 1/2, p. 91-106.

ALLEN, Sarah, et Kerry DALY (2007). Les effets de l'engagement paternel : version actualisée d'un résumé de données de recherche, Université de Guelph, $50 \mathrm{p}$.

BASHAM, Kathryn (2008). "Homecoming as Safe Haven or the New Front: Attachment and Detachment in Military Couples ", Clinical Social Work Journal,Vol. 36, p. 83-96.

BASS, Brenda L., et collab. (2009). "Do Job Demands Undermine Parenting? A Daily Analysis of Spillover and Crossover Effects ", Family Relations, Vol. 58, p. 201-215.

BEDFORD, Nicole (2009). "On discute de l'intégration des femmes au sein des FC ", Les Nouvelles de l'Armée, réf. du 20 mars 2010, http://www.army.dnd.ca/land-terre/news-nouvelles/texttexte-fra.asp?id $=3342$.

BELIK, Shay-Lee, et collab. (2009). «Relation between Traumatic Events and Suicide Attempts in Canadian Military Personel », Canadian Journal of Psychiatry, Vol. 54, № 2, p. 93-104.

BIELBY, William T., et Denise D. BIELBY (1992). «I will Follow him: Famili Ties, Gender-Role Beliefs, and Reluctance to Relocate for a Better Job ", The American Journal of Sociology, Vol. 97 , $\mathrm{N}^{\circ}$ 5, p. 1241-1267. 
BOWEN, Gary L., et collab. (2003). "Promoting the Adaptation of Military Families:An Empirical Test of a Community Practice Model ", Family Relations, Vol. 52, No 1, p. 33-44.

BOYLE, P., et collab. (2001). "A cross-national comparison of the impact of family migration on women's employment status ", Demography, Vol. 38, p. 201-213.

BRIGGS, Andrew, et Pam ATKINSON (2006). "Adapting the Model: Therapeutic Work with Children from Army Families ", Journal of Social Work Practice, Vol. 20, № 1, p. 51-67.

BROOKS, Gary R. (1998). A New Psychotherapy for Traditional Men, San Francisco : Jossey-Bass Publishers, $262 \mathrm{p}$.

BUSUTTIL, Walter, et Angela BUSUTTIL (2001). " Psychological effects on families subjected to an enforced and prolonged separations generated under life threatening situations ", Sexual and Relationship Therapy. Vol. 16, № 3, p. 207-228.

CANADA, MINISTÈRE DE LA DÉFENSE NATIONALE (2009). Données démographiques sur les Forces canadiennes de 2009, Directeur - Gestion de l'information des ressources humaines des Forces canadiennes, Document interne : le 15 octobre 2009, № 5390-2 (DGSSPF).

CANADA, MINISTÈRE DE LA DÉFENSE NATIONALE (2008a). Formation des recrues, réf. du 20 mars 2010, http://www.forces.ca/\#fr.

CANADA, MINISTÈRE DE LA DÉFENSE NATIONALE (2008b). Services de santé des Forces canadiennes : le système de santé des militaires, réf. du 20 mars 2010, http://www.forces.gc.ca/ health/frgraph/home_f.asp.

CANADA, MINISTÈRE DE LA DÉFENSE NATIONALE (2008c). Engagement des Forces canadiennes à l'endroit des familles, réf. du 20 mars 2010, https://www.cfpsa.com/en/psp/DMFS/pdfs/CF\%20 Family\%20Covenant.pdf.

CANADA, MINISTÈRE DE LA DÉFENSE NATIONALE (2008d). Une carrière en tant qu'officier : Travail social, réf. du 20 mars 2010, http://www.forces.ca/media/_PDF/58_fr.pdf.

CANADA, MINISTÈRE DE LA DÉFENSE NATIONALE (2006). The National Defence Official Language Transformation Model, Document interne : le 25 octobre 2006.

CANADA, MINISTÈRE DE LA DÉFENSE NATIONALE (2003). Énoncé d'éthique de la défense, réf. du 20 mars 2010, http://www.admfincs.forces.gc.ca/admfincs/subjects/daod/7023/ form/a_f.asp.

CANADIAN FORCES HEALTH SERVICES GROUP (2010). Report of the Canadian Forces Expert Panel on Suicide Prevention, Défense nationale, Report number 2010-01, 80 p.

CARAGATA, Lea, et Wayne MILLER (2008). What Supports Engaged Fathering? Employment and Family Supports, FIRA (Pub.), 43 p.

COMMISSARIAT AUX LANGUES OFFICIELLES (2007). "Forces canadiennes : Fiche d'information 2005-2006 ", Bulletins de rendement, réf. du 17 février 2010, http://www.ocol-clo. gc.ca/html/dnd_mdn_05_06_f.php.

CORRIVEAU, Claire (2007). Les épouses de l'armée, [vidéo], Office national du Film, 52 minutes.

COTTON, Charles A. (1981). "Institutional and Occupational Values in Canada's Army ", Armed Forces and Society, Vol. 8, № 1, p. 99-110.

CREAMER, Mark, et David FORBES (2004). «Treatment of Posttraumatic Stress Disorder in Military and Veteran Populations ", Psychotherapy : Theory, Research, Practice, Training, Vol. 41, № 4, p. 388-398. 
CENTRE DE RESSOUCES FAMILLE-MILITAIRE DE VALCARTIER (2009). Présentation du centre, réf. du 20 mars 2010, http://www.crfmv.com/arrivants-presentation.php.

DALEY, James G. (2003). « Military social work », International Social Work, Vol. 46, No 4, p. 437448.

DARWIN, Jaine L., et Kenneth I. REICH (2006). « Reaching Out to the Families of Those Who Serve :The SOFAR Project ", Professional Psychology: Research et Practice, Vol. 37, No 5, p. 481484.

DAVIES, L., et collab. (2009). "Une place pour les pères : un nouveau paradigme dans les pratiques sociales en petite enfance ", Reflets,Vol. 15, p. 38-59.

DESLAURIERS, Jean-Martin (2009). "Paternités, discours et pratiques sociales : quelles passerelles possibles? ", Reflets : revue d'intervention sociale et communautaire, Vol. 15, No 1, p. 10-18.

DIRKZWAGER, Anja J. E., et collab. (2005). "Secondary Traumatization in Partners and Parents of Dutch Peacekeeping Soldiers ", Journal of Family Psychology, Vol. 19, № 2, p. 217-226.

DRUMMET, Amy Reinkober, Marilyn COLEMAN et Susan CABLE (2003). " Military Families Under Stress: Implications for Family Life Education ", Family Relations, Vol. 52, № 3, p. 279287.

DUBAR, Claude (2000). La socialisation : Construction des identités sociales et professionnelles, Paris : Armand Colin, $278 \mathrm{p}$.

DUBEAU, Diane, Annie DEVAULT et Gilles FOR GET (2009). Prospère : La paternité du $21^{\text {ème }}$ siècle, Presses de l'Université Laval.

DUBEAU, Diane, Marie-Ève CLÉMENT et Claire CHAMBERLAND (2005). « Le père, une roue du carrosse familial à ne pas oublier! État des recherches québécoises et canadiennes sur la paternité », Enfances, Familles, Générations, no 3, réf. du 20 mars 2010, http://www.erudit.org. proxy.bib.uottawa.ca/revue/efg/2005/v/n3/012534ar.html.

DULAC, Germain (2009). "La conciliation travail-famille : Le point de vue des pères ", Pères, No 6, p. 65-98.

FEATHERSTONE, Brid (2004). "Fathers Matter: A Research Review ", Children et Society, Vol. 18, p. 312-319.

FIGLEY, Charles E. (2005). « Strangers at Home: Comment on Dirkswager, Bramsen, Adèr, andVan der Ploeg (2005) ", Journal of Family Psychology, Vol. 19. № 2, p. 227-229.

FLOURI, Eirini, et Ann BACHANAN (2003). "The Role of Father Involvement in Children's Later Mental Health », Journal of Adolescence, Vol. 26, № 1, p. 63-78.

FOR GET, Gilles (2009). "La promotion de l'engagement paternel, des archétypes à transformer, une pratique à construire ", Reflets : revue d'intervention sociale et communautaire, Vol. 15, No 1, p. 79-101.

GOFFMAN, Erving (1968). Asiles - Études sur la condition sociale des malades mentaux et autres reclus, Paris, Éditions de Minuit.

GROSSMAN, Dave (1995). On Killing : The Psychological Cost of Learning to Kill in War and Society, Boston, Little, Brown and Company.

HARRISON, Deborah (2006). "The Role of Military Culture in Military Organizations' Response to Woman Abuse in Military Families ", The Sociological Review, Vol. 54, No 3, p. 546-574.

HARRISON, Deborah (2002). The First Casualty : Violence against Women in Canadian Military Communities, Toronto : James Lorimier et Co., 256 p. 
HARRISON, Deborah (1997). «La violence dans la communauté militaire », Criminologie,Vol. 30, $\mathrm{N}^{\circ} 2$, p. 27-45.

HARRISON, Deborah, et Lucie LALIBERTÉ (1997). "Gender, the Military, and Military Family Support ", dans L.Weinstein, et C. C. White (Eds.), Wives et Warriors : Women and the Military in the United States and Canada, Westport, Connecticut : Bergin et Garvey, chapter 3, p. 35-53.

HIEW, Chok C. (1992). «Seperated by their Work: Families with Fathers Living Apart ", Environment and Behavior,Vol. 24, № 2, p. 206-225.

HORTON, Denise (2005). "Consultation with Military Children and Schools :A Proposed Model», Consulting Psychology Journal: Practice and Research,Vol. 57, No 4, p. 259-265.

HUEBNER, Angela J., et collab. (2007). "Parental Deployment and Youth in Military Families : Exploring Uncertainty and Ambiguous Loss ", Family Relations, Vol. 56, № 2, p. 112-122.

JELUSIC, Ljubica (2005). « Ritualization of Emotions in Military Organization », Military Sociology, Eric Ouellet (Ed.), Whitby, Ontario : de Sitter Publications, Chapter 8, p. 230-254.

JÜR GES, Hendrik (2006). «Gender Ideology, Division of Housework, and the Geographic Mobility of Families ", Review of Economics in the Household,Vol. 4, p. 299-323.

KAHN, Jack S. (2009). An Introduction to Masculinities, West Sussex, UK: Wiley-Blackwell, 432 p.

LAMB, Michael (2000). "The History of Research on Father Involvment : An Overview ", Marriage and Family Review, Vol. 29, № 2/3, p. 23-42.

LEVANT, Ronald F., et William S. POLLACK (1995). A New Psychology of Men, New York : Basic Books, $402 \mathrm{p}$.

MACÉ, Éric (2002). "L'exigence de sécurité, une question politique ", État, société et délinquance : Cahiers français, $\mathrm{N}^{\circ} 308$, p. 1-5.

MER CIER, Peter J. (2000). Battle Cries on the Home Front: Violence in the Military Family, Peter J. Mercier et Judith D. Mercier (Eds.), Springfield, IL. : Charles C. Thomas Publisher, 220 p.

PARK, Jungwee (2008). «A profile of the Canadian Forces ", Perspectives, Statistics Canada - Catalogue $\mathrm{N}^{\mathrm{o}}$ 75-001-X, p. 17-30.

PINCUS, Simon H., et collab. (2008). «The Emotional Cycle of Deployment: A Military Family Perspective ", My Hooah 4 Health, réf. du 20 mars 2010, http://www.hooah4health.com/ deployment/Familymatters/emotionalcycle.htm.

PITTMAN, Joe F., Jennifer L. KERPELMAN et Jennifer M. McFADYEN (2004). "Internal and External Adaptation in Army Families : Lessons from Operations Desert Shield and Desert Storm ", Family Relations, Vol. 53, № 3, p. 249-260.

ROCHER, Guy (1992). Introduction à la sociologie générale : 1. L'action sociale, Lasalle, Qc : Hurtubise HMH, $190 \mathrm{p}$.

ROTTER, Joseph C., et Marcha E. BOVEJA (1999). "Counselling Military Families », The Family Journal: Counselling and Therapy for Couples and Families,Vol. 7, No 4, p. 379-382.

RYAN-WENGER, Nancy A. (2001). "Impact of the Threat of War on Children in Military Families ", American Journal of Orthopsychiatry, Vol. 71, No 2, p. 236-244.

SCHEHR, Sébastien (2005). "Le quotidien comme miroir de l'individualisation de la condition militaire : pistes de recherche et perspectives méthodologiques ", dans Sociologie du milieu militaire : Les conséquences de la professionnalisation sur les Armées et l'identité militaire, François Gresle (Éd.). Paris : L'Harmattan, chapitre 10, p. 205-228. 
SERVICES DE SOUTIEN AU PERSONNEL ET AUX FAMILLES DES FORCES MILITAIRES CANADIENNES (2010). Programme des services aux familles des militaires, réf. du 20 mars 2010, http://www.cfpsa.com/fr/psp/dmfs/about.asp.

SMITH, Mark (2004). "What about the Dads? Issues and Possibilities of Working with Men from a Child and Youth Care Perspective ", Child and Youth Services,Vol. 25, № 1, p. 149-167.

STROBINO, Jane, et Mary SALVATERRA (2000). «School Transitions among Adolescent Children of Military Personnel: A Strenghts Perspective ", Social Work in Education, Vol. 22, No 2, p. 95 107.

TARDIF, Claudette, et Marie-Joie BRADY (2009). "Les droits linguistiques au Canada : un minimalisme inquiétant ", Le caucus libéral du Sénat du Canada, réf. du 20 mars 2010, http:// www.forumdessenateursliberaux.ca/Dans-le-Senat/Publication/2766_Les-droits-linguistiquesau-Canada--un-minimalisme-inquietant.

TREMBLAY, Diane-Gabrielle, Charles-Henri AMHERDT et Monique K. De SÈVE (2003). La conciliation emploi-famille : des mesures à développer dans les milieux de travail. Résultats d'enquête auprès de sept organisations rattachées au syndicat canadien de la fonction publique, Chaire de recherche sur les enjeux socio-organisationnels de l'économie du savoir, no de recherche 2005-05, 157p.

TRUSCOTT, Susan, et Kate DUPRE (1998). Organizational, Social, and Demographic Change in the Canadian Forces, Department of National Defence, Canada, AB-37-Paper, réf. du 20 mars 2010, http://www.internationalmta.org/1998/9837.html.

VAN DER KOLK, Bessel, OnnoVAN DER HART et Jenifer BURBRIDGE (2002). «Approaches to the Treatment of PTSD ", in Simple and Complex Post-Traumatic Stress Disorder : Strategies for Comprehensive Treatment in Clinical Practice, Mary Beth Williams et John F. Sommers Jr. (Eds.). New York : Haworth Maltreatment and Trauma Press, chapter 3, p. 23-45.

VERSCHELDEN, Cia (1993). "Social WorkValues and Pacifism : Opposition to War as a Professional Responsibility ", Social Work,Vol. 38, № 6, p. 765-769.

VILLENEUVE, Raymond (2009). «Réflexions du Regroupement pour la valorisation de la paternité sur le rapport du conseil de la famille et de l'enfance portant sur l'engagement des pères ", Reflets : revue d'intervention sociale et communautaire, Vol. 15, N $\mathrm{N}^{\circ} 1$, p. 216-224.

VOYDANOFF, Patricia (2004). «The Effects of Work and Community Resources and Demands on Family Integration ", Journal of Family and Economic Issues, Vol. 25, No 1, p. 7-23.

WINSLOW, Donna, et Jason DUNN (2002). «Women in the Canadian Forces : Between Legal and Social Integration ", Current Sociology, Vol. 50, No 5, p. 641-667. 